\title{
Revealing Local Field Structure of Focused Ultrashort Pulses
}

\author{
Cyril Hnatovsky, ${ }^{1}$ Vladlen Shvedov, ${ }^{1,2}$ Wieslaw Krolikowski, ${ }^{1}$ and Andrei Rode ${ }^{1}$ \\ ${ }^{1}$ Laser Physics Center, Research School of Physics and Engineering, Australian National University, Canberra ACT 0200, Australia \\ ${ }^{2}$ Nonlinear Physics Center, Research School of Physics and Engineering, Australian National University, \\ Canberra ACT 0200, Australia
}

(Received 16 December 2010; revised manuscript received 1 March 2011; published 23 March 2011)

\begin{abstract}
We utilize the interaction of tightly focused ultrashort pulses with transparent media to imprint their local polarization in the focal region. In particular, we demonstrate that this technique allows for a subwavelength resolution diagnostic of complex polarization states including the presence of the longitudinal component of the electric field. Moreover, we demonstrate the first ever material ablation with the longitudinal electric field of femtosecond pulses.
\end{abstract}

PACS numbers: 42.25.Ja, 42.60.Jf, 42.65.Re, 79.20.Eb

Polarization is one of the most important properties of light affecting its interaction with matter. Until the early 1970s, most of the related work concentrated on spatially homogeneous states of light polarization. The advent of lasers generating bright coherent light beams allowed researchers to impose complex polarization structure on light fields in order to expand functionality of the existing optical techniques and investigate new phenomena [1]. For example, radially polarized beams focused with high numerical aperture (NA) optics are now routinely used in microscopy [2-4], Raman spectroscopy [5], secondharmonic generation [6], and particle trapping and manipulation [7] where the presence of a strong longitudinal component (the $z$ component) of the electric field in a very tight laser focus is crucial for these applications [8]. In general, for any technique requiring subwavelength field distributions, the vectorial character of light becomes critical [9] so that most of the recent studies of highly focused optical beams have mainly concentrated on their three-dimensional intrafocal polarization structure [10]. Observation of nonparaxial polarization effects on a nanoscale is a nontrivial task requiring refined experimental setups and usually a priori knowledge of the expected field distribution. The intrafocal mapping of the squared electric field components is usually achieved with near field probes [11], point scatterers [12], fluorescent molecules and beads [2,13], and the knife edge method [14]. Only a few representatives of broad classes of cylindrical vector and singular beams have been studied experimentally so far $[13,14]$.

Here we present an entirely new approach to visualize nanoscale structure of the electric field of tightly focused ultrashort laser pulses by permanently imprinting the field, including its longitudinal component, in transparent media. We show, for the first time, that the ensuing nanopatterns accurately replicate local polarization structure of a femtosecond light beam and, conversely, behave as birefringent nanostructures. The demonstrated local character of the light-matter interaction allows one to perform subwavelength resolution polarization diagnostics (and control) of the focal volume of tightly focused ultrashort pulse laser beams with complex vectorial structure which are becoming increasingly important in a number of applications $[4,6,15]$.

Studies on the interaction of high-energy ultrashort laser pulses with optically transparent media have led to the discovery of two major classes of phenomena. It has been shown that the interaction of single pulses with matter is essentially an intensity-dependent process and the ensuing material modification bears no signature on the light polarization. On the other hand, the response of some materials to multiple pulse irradiation depends on both the intensity and polarization of the light field and leads to structured changes in the material. To reveal the complex polarization state of tightly focused light, we use the ability of laser radiation to produce self-organized nanostructures on liquid and solid surfaces and inside transparent materials. The surface phenomenon, i.e., laser-induced periodic surface structures or surface ripples, was first reported in 1965 [16], and since then laser-induced periodic surface structures have been observed in a variety of materials including dielectrics, semiconductors, and metals under widely different illumination conditions [17]. Continuous and pulsed (including subpicosecond) laser radiation at wavelengths ranging from the middle infrared to the near ultraviolet was used in these experiments. With rare exceptions, e.g., $[18,19]$, studies on this surface phenomenon have been performed with linearly polarized light, which leads to the formation of one-dimensional gratinglike structures. Much later, in 2003, Shimotsuma et al. [20] demonstrated that when glass is irradiated with a series of focused linearly polarized femtosecond laser pulses, subwavelength gratinglike distribution of the refractive index can be created around the focal region inside the material. The bulk nanograting, similar to the majority of laser-induced periodic surface structures [17], tends to align itself perpendicular to the electric field of the light wave. This observation, however, is based on experiments 
performed solely with linearly polarized light, and until now it has not been clear how the material responds to high-intensity laser pulses with complex local polarization structure. Under certain irradiation conditions the nanograting evolves into an array of planar nanocracks [21,22]. There is evidence that circularly polarized femtosecond light focused into fused silica glass produces a permanent recording of optical helicity in the form of highly ordered submicron chiral structures whose handedness follows the handedness of the circularly polarized light [23]. So far, the formation of bulk nanogratings has been observed only with subpicosecond pulses. Even though the mechanism which governs the formation of the bulk gratings remains to be ascertained $[20,24]$, these structures are routinely produced inside transparent media and can find applications in 3D rewritable data storage in glass, embedded micro-optic arrays, and microfluidics [22].

The sensitivity of materials' response to the state of polarization of high-intensity light fields has been used in our experiments to map the complex vectorial structure of the focal volume of cylindrical, i.e., radial (TM), azimuthal (TE), and mixed polarization femtosecond laser beams. To generate TM and TE pulses we first form single-charge circularly polarized vortex pulses by using a step phase mask and a quarter wave plate and ensure that the polarity $m$ and the handedness $\sigma$ of the vortex are of opposite signs (i.e., $\sigma= \pm 1, m=\mp 1$ ) [25]. The electric field of such a vortex is a superposition of a TM and a TE mode [26].

Then we let the vortex propagate along the optical axis of a uniaxial crystal which is placed between a negative lens L1 and a positive lens L2 [Fig. 1]. TM and TE fields are eigenmodes of a uniaxial crystal with their waists being axially shifted with respect to each other due to double

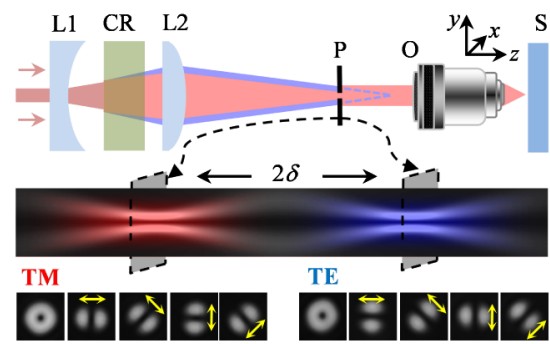

FIG. 1 (color online). Beam converter for the synthesis of radially (TM), azimuthally (TE), and mixed polarization femtosecond pulses. L1 denotes a negative $(-50 \mathrm{~mm})$ fixed lens and L2 a positive $(+125 \mathrm{~mm})$ movable lens, CR is a 10-mm-long, $c$-cut calcite crystal, $P$ is a pinhole $300 \mu \mathrm{m}$ in diameter located $150 \mathrm{~cm}$ away from L2, $O$ is a focusing objective, and $S$ is a glass sample. The beam waist in the pinhole plane is $\approx 100 \mu \mathrm{m}$. Red and blue represent schematically the TM and TE modes, respectively. The axial shift of $\mathrm{L} 2$ and $P$ to discriminate the TM and TE modes is $2 \delta=1.44 \mathrm{~mm}$. CCD images of the generated TM and TE modes together with the corresponding intensity distributions after an analyzer whose transmission axis is denoted by arrows are shown at the bottom. refraction in the crystal. For paraxial beams the shift is approximated by $2 \delta=\left(n_{o}^{2}-n_{e}^{2}\right) /\left(n_{e}^{2} n_{0}\right)$, where $n_{o}$ and $n_{e}$ stand for the ordinary and extraordinary refractive index, respectively, and $d$ is the thickness of the crystal along the beam propagation direction $z$.

The azimuthally and radially polarized pulses are discriminated by shifting the lens L2 together with the pinhole $P$ along the $z$ axis by $2 \delta$ and thus placing the respective waists of either the TE or TM beams in the pinhole plane [see Fig. 1]. After the pinhole the beam is collimated and directed to the focusing optics (viz., NA $=0.9$ Nikon MPlan $100 \times$ or $\mathrm{NA}=0.46$ Olympus MSPlan $20 \times$ ). Theoretical analysis shows that for our experimental conditions the resulting mode purity exceeds $98 \%$ [25]. In our experiments we used the output beam of a Clark-MXR femtosecond Ti:sapphire amplifier with a central wavelength at $\lambda \approx 775 \mathrm{~nm}$. After the lens L2, the pulse duration is estimated at $\approx 200 \mathrm{fs}, \mathrm{FWHM}$, based on noncollinear autocorrelation measurements. To correct for spherical aberration which is introduced when finite conjugate distance optics is used for focusing collimated beams and to minimize temporal stretching of the pulses by the focusing optics, a corrector comprised of a negative $(-200 \mathrm{~mm})$ lens followed by an opaque stop $\left(2 R_{1}=2.2 \mathrm{~mm}\right.$ in diameter) is placed near the entrance aperture of the $\mathrm{NA}=0.9$ objective [14,27].

Shown in Fig. 2 are the salient features of the material's response to single and multiple femtosecond pulses with
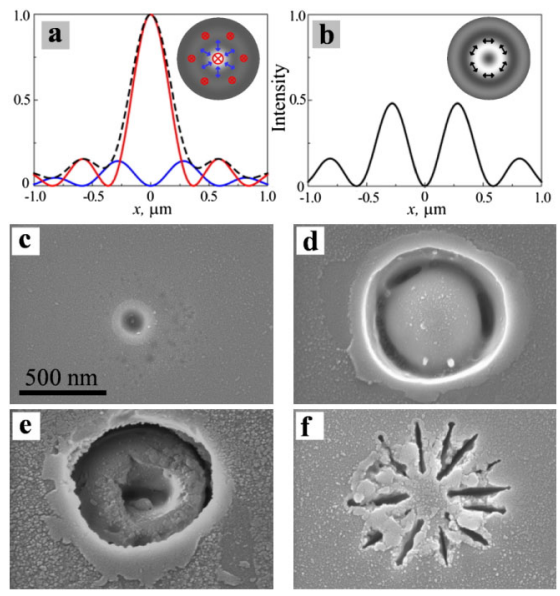

FIG. 2 (color online). (a),(b) Simulated intensity distributions in vacuum of a radially and azimuthally polarized beam, respectively, in the focal plane of a NA $=0.9$ objective. In (a), the intensity of the longitudinal (i.e., $z$ component) and transverse (i.e., radial) component is shown in red and blue, respectively, and the total intensity is represented by a dashed line. Scanning electron microscopy images of borosilicate Corning 0211 glass samples' response to (c) the longitudinal component of a $30 \mathrm{~nJ}$ single TM pulse; (d) the transverse (i.e., azimuthal) component of a $120 \mathrm{~nJ}$ single TE pulse; (e) irradiation with multiple $80 \mathrm{~nJ}$ TM pulses; and (f) irradiation with multiple $40 \mathrm{~nJ}$ TE pulses. The scale bar is the same for (c)-(f). 
different states of polarization focused with the NA $=0.9$ objective. The simulated intensity distributions in vacuum for the longitudinal $(z)$ and transverse electric field components in the focal plane are presented in Figs. 2(a) and 2(b). The simulations are based on the Debye-Wolf integral with a Laguerre-Gaussian amplitude function $[9,28,29]$. One can show that the amplitude of the electric field of radially $\left(\vec{E}_{\mathrm{TM}}\right)$ and azimuthally $\left(\vec{E}_{\mathrm{TE}}\right)$ polarized beams in the focal area of an objective can be written as $\vec{E}_{\mathrm{TM}}=4 \pi\left(i I_{r} \vec{e}_{r}-2 I_{0} \vec{e}_{z}\right)$ and $\vec{E}_{\mathrm{TE}}=8 \pi I_{\phi} \vec{e}_{\phi}$, where

$$
\begin{aligned}
I_{r} & =\frac{-i A}{2 \lambda} \int_{\alpha_{1}}^{\alpha} A_{1}(\theta) \sqrt{\cos \theta} J_{1}\left(k r_{p} \sin \theta\right) e^{-i k z \cos \theta} \sin 2 \theta d \theta, \\
I_{\phi} & =\frac{-i A}{2 \lambda} \int_{\alpha_{1}}^{\alpha} A_{1}(\theta) \sqrt{\cos \theta} J_{1}\left(k r_{p} \sin \theta\right) e^{-i k z \cos \theta} \sin \theta d \theta, \\
I_{0} & =\frac{-i A}{2 \lambda} \int_{\alpha_{1}}^{\alpha} A_{1}(\theta) \sqrt{\cos \theta} J_{0}\left(k r_{p} \sin \theta\right) e^{-i k z \cos \theta} \sin ^{2} \theta d \theta,
\end{aligned}
$$

and $\theta$ is the angular coordinate, $\alpha$ stands for the numerical aperture of the objective, $\alpha_{1}$ denotes the angular dimension of the circular beam stop of radius $R_{1}$ which blocks the central part of the beam, $A_{1}(\theta) \propto \sqrt{2} \gamma \sin \theta / \sin \alpha \times$ $\exp \left[-(\gamma \sin \theta / \sin \alpha)^{2}\right]$ describes the angular amplitude distribution of the collimated incident (i.e., TM or TE) beam, constant $A$ is related to the parameters of the optical system, $k=2 \pi / \lambda$ is the wave number of light in the medium with refractive index $n, r_{P}$ is the radial distance of point $P$ from the $z$ axis, $J_{m}\left(k r_{p} \sin \theta\right)$ denotes a Bessel function of the first kind of order $m$, and the truncation parameter $\gamma=R / w$ is the ratio of the entrance aperture of radius $R$ and the beam size parameter $w$. In our experiments, $R_{1}=1.1 \mathrm{~mm}, R=2 \mathrm{~mm}, n=1, \alpha=0.9$, and $\alpha_{1}=0.75$. The integral was calculated only for the central wavelength $(\lambda \approx 775 \mathrm{~nm})$ of the femtosecond laser radiation which has a FWHM spectral bandwidth of $\approx 5 \mathrm{~nm}$. The effect of light polychromaticity on the intrafocal intensity distribution becomes noticeable only for very short $(<10 \mathrm{fs})$, broadband laser pulses [30]. However, even in this extreme case the main features associated with the vectorial character of the beam remain essentially intact.

The presence of a strong longitudinal electric field component in TM pulses is evidenced by a less than $100 \mathrm{~nm}$ wide ablation crater at the focal axis [Fig. 2(c)]. Note that the transverse component of the electric field is identically zero in the center, and hence the crater can be produced only by the action of the longitudinal field component. In fact, these results constitute the first demonstration of material ablation with the longitudinal component of the electric field of an optical beam. On the other hand, single TE pulses [Fig. 2(d)] produce only ring-shaped ablation signatures without any features at the center. These observations confirm that in the single pulse irradiation mode the material responds to the intensity distribution in the focus and the ensuing material modification bears no information on the polarization state of the light. The outcome of laser-matter interaction is more dramatic when the same spot is irradiated by $\approx 10^{3}$ pulses. In this regime, the material becomes sensitive to both the intensity and polarization of the laser field. Multiple TM pulses now produce a central crater surrounded by a circular deep crack [Fig. 2(e)]. Again, the crater is caused by the action of the longitudinal component of the electric field, whereas the crack is produced by the transverse (i.e., radial) component. On the other hand, TE pulses generate cracks which are aligned radially, strictly normal to the direction of the electric vector at each point [Fig. 2(f)]. It should be emphasized that the observed cracks are micron deep and are therefore related to the bulk phenomenon mentioned in the introduction.

This technique enables, in principle, any intrafocal vectorial field configurations to be imprinted inside the material [25]. For instance, we used the same beam converter to synthesize light vector field states with polarization structure shown in Figs. 3(a) and 3(b). These states are formed by using linearly polarized input Gaussian beam, and the two foci are discriminated exactly in the same manner as for the TM and TE modes. The resulting vector maps shown in Figs. 3(a) and 3(b) are, in fact, very similar, because in the areas where the light intensity is strong both fields are essentially linearly polarized and only in the peripheries do the lines of polarization vectors exhibit different curvatures. In the first set of experiments laser pulses were focused by using a microscope objective of
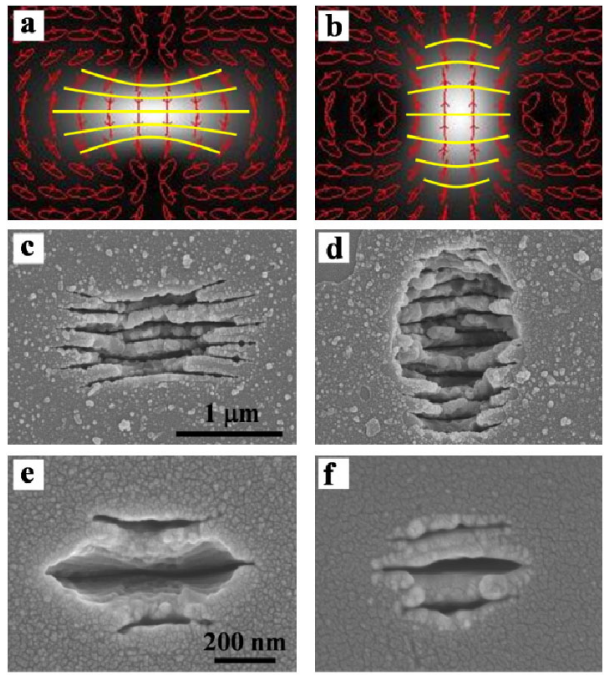

FIG. 3 (color online). Imprinting the electric field structure of a mixed polarization femtosecond pulse in fused silica. (a),(b) Simulated electric vector maps after the beam converter. (c),(d) Imprints produced with a focusing objective with an effective NA of $\approx 0.3$ after irradiation with multiple $200 \mathrm{~nJ}$ pulses. (e),(f) Imprints produced with a NA $=0.9$ focusing objective after irradiation with multiple $25 \mathrm{~nJ}$ pulses. The scale bars shown in the left column apply to the corresponding images in the right column. The expected orientation of the nanocracks is denoted in (a),(b) with yellow lines. 

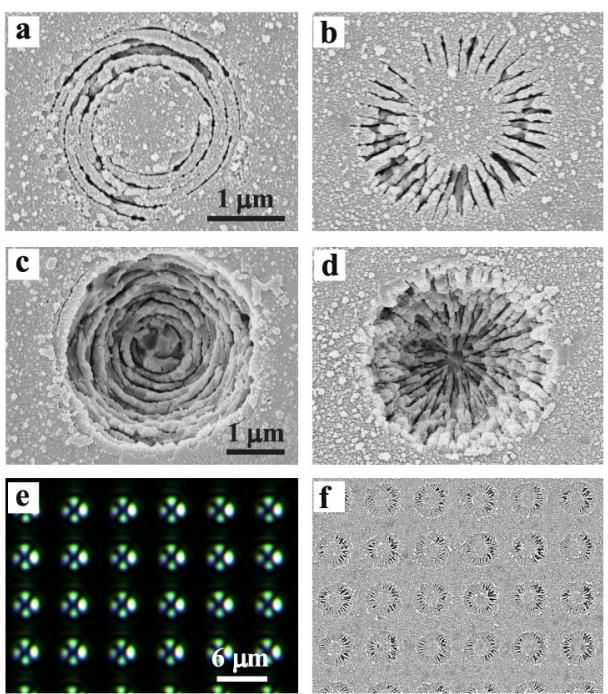

FIG. 4 (color online). Polarization-sensitive micro-optic structures produced inside fused silica substrates with radially (TM) and azimuthally (TE) polarized femtosecond pulses. (a), (b) Imprints produced with a focusing objective with an effective NA of $\approx 0.3$ after irradiation with multiple $300 \mathrm{~nJ}$ pulses. (c), (d) The same conditions as in (a),(b) except that the pulse energy is $500 \mathrm{~nJ}$. (e) Optical signature of the two-dimensional array of imprints depicted in (f) as seen in cross-polarized light. The scale bars shown in the left column apply to the corresponding images in the right column.

moderate NA (viz., NA $=0.46$ ). In this case the incident beam underfilled the entrance aperture of the objective, which reduced its nominal NA to $\approx 0.3$. From Figs. 3(c) and $3(\mathrm{~d})$, one can see that the light-induced cracks accurately follow subtle variations in the field structure within a micron size focus. In the regime of a very tight focusing (viz., NA $=0.9$ ) the field structure can still be visualized by nanocracks even though only three of them are produced in this case [Figs. 3(e) and 3(f)].

Figure 4 shows the structure of cracks produced with the $\mathrm{NA}=0.46$ objective in fused silica samples. In the cases shown in Figs. 4(a) and 4(b) the patterns are produced near the threshold pulse energy, whereas in Figs. 4(c) and 4(d) the pulse energy was increased and significant material ablation occurred. The morphology of the ablation craters provides insight into the 3D structure of the nanocrack patterns and, consequently, the beam polarization state. Notice perfectly visible azimuthal [Fig. 4(c)] and radial [Fig. 4(d)] nanocrack patterns reflecting the TM and TE character of the field, respectively. It is also noteworthy that patterns recorded with moderately focused TM pulses show that the longitudinal component of the electric field is too weak in this case to produce any material modification, in agreement with the theory. When embedded in bulk material these polarization imprints can be easily observed and analyzed in cross-polarized light as shown in Fig. 4(e) due to strong form birefringence of the nanopatterns [22,31]. Such structures may find applications in the fabrication of polarization micro-optic components and beam shapers, in polarization-encoded multilevel optical data storage, and in the synthesis of chiral materials [25].

We thank Dr. F. Brink and Dr. H. Chen for help with the scanning electron microscopy images and N. Shostka for modeling of polarization maps. This work was supported by the National Health and Medical Research Council of Australia and the Australian Research Council.

[1] M. R. Dennis, K. O'Holleran, and M. J. Padgett, Prog. Opt. 53, 293 (2009).

[2] L. Novotny et al., Phys. Rev. Lett. 86, 5251 (2001).

[3] K. Watanabe, N. Horiguchi, and H. Kano, Appl. Opt. 46, 4985 (2007).

[4] E. Y.S. Yew and C. J. R. Sheppard, Opt. Commun. 275, 453 (2007).

[5] N. Hayazawa, Y. Saito, and S. Kawata, Appl. Phys. Lett. 85, 6239 (2004).

[6] D. P. Biss and T. G. Brown, Opt. Lett. 28, 923 (2003).

[7] Q. Zhan, Opt. Express 12, 3377 (2004).

[8] H. Wang et al., Nat. Photon. 2, 501 (2008).

[9] B. Richards and E. Wolf, Proc. R. Soc. A 253, 358 (1959).

[10] Q. Zhan, Adv. Opt. Photon. 1, 1 (2009).

[11] S. K. Rhodes, K. A. Nugent, and A. Roberts, J. Opt. Soc. Am. A 19, 1689 (2002).

[12] T. Wilson, R. Juškaitis, and P. Higdon, Opt. Commun. 141, 298 (1997).

[13] N. Bokor et al., Opt. Express 13, 10440 (2005).

[14] R. Dorn, S. Quabis, and G. Leuchs, Phys. Rev. Lett. 91, 233901 (2003).

[15] B. Jia et al., Opt. Lett. 34, 1918 (2009).

[16] M. Birnbaum, J. Appl. Phys. 36, 3688 (1965).

[17] D. Bäuerle, Laser Processing and Chemistry (Springer, Berlin, 2000), 3rd ed.

[18] F. Keilmann and Y. H. Bai, Appl. Phys. A 29, 9 (1982).

[19] O. Varlamova et al., Appl. Surf. Sci. 252, 4702 (2006).

[20] Y. Shimotsuma et al., Phys. Rev. Lett. 91, 247405 (2003).

[21] C. Hnatovsky et al., Appl. Phys. Lett. 87, 014104 (2005).

[22] R. S. Taylor, C. Hnatovsky, and E. Simova, Laser Photon. Rev. 2, 26 (2008).

[23] R. S. Taylor, E. Simova, and C. Hnatovsky, Opt. Lett. 33, 1312 (2008).

[24] V. R. Bhardwaj et al., Phys. Rev. Lett. 96, 057404 (2006).

[25] See supplemental material at http://link.aps.org/ supplemental/10.1103/PhysRevLett.106.123901 for the detailed calculations pertaining to the beam converter and for the images of imprints produced with spirally polarized fields.

[26] T. Fadeyeva et al., Opt. Lett. 35, 3787 (2010).

[27] M. Kempe, U. Stamm, and B. Wilhelmi, Opt. Commun. 89, 119 (1992).

[28] K. S. Youngworth and T. G. Brown, Opt. Express 7, 77 (2000).

[29] P. Török and P. Munro, Opt. Express 12, 3605 (2004).

[30] K. M. Romallosa, J. Bantang, and C. Saloma, Phys. Rev. A 68, 033812 (2003).

[31] M. Born and E. Wolf, Principles of Optics (Pergamon, Oxford, 1980), 6th ed. 